\title{
HEAT KERNEL TRANSFORM ON NILMANIFOLDS ASSOCIATED TO H-TYPE GROUPS
}

\author{
Aparajita Dasgupta AND Sundaram ThangaVElu
}

(Received July 7, 2010, revised January 30, 2012)

\begin{abstract}
We study the heat kernel transform on a nilmanifold $\Gamma \backslash N$ associated to an $\mathrm{H}$-type group. Using a reduction technique we reduce the problem to the case of Heisenberg groups. The image of $L^{2}(\Gamma \backslash N)$ under the heat kernel transform is shown to be a direct sum of weighted Bergman spaces.
\end{abstract}

1. Introduction. The aim of this article to study the heat kernel transform on nilmanifolds associated to nilpotent groups of Heisenberg type (H-type in short). Heat kernel transform on the Heisenberg group $\boldsymbol{H}^{n}$ and the nilmanifold associated to the standard lattice $\Gamma=\boldsymbol{Z}^{n} \times \boldsymbol{Z}^{n} \times(1 / 2) \boldsymbol{Z}$ has been studied by Krötz-Thangavelu-Xu in [5] and [6], respectively. Here we extend their results to the general nilmanifold associated to an H-type group.

The problem on H-type groups can be easily reduced to the case of $\boldsymbol{H}^{n}$ via a partial Radon transform. This technique employed by Ricci [11], Müller [8] and others has turned out to be very useful. Thus given a nilmanifold on an H-type group, the problem can be reduced to the study of a family of Heisenberg nilmanifolds. Lattice subgroups of the Heisenberg group leading to nilmanifolds have been completely characterised, thanks to the work of Tolimieri [14]. Up to an automorphism, coming from the symplectic group, they are given by lattices of the form $\Gamma$ (I) with $\mathbf{I}=\left(l_{1}, \ldots, l_{n}\right)$ where $l_{j}$ 's are positive numbers such that $l_{j}$ divides $l_{j+1}$ (see next section).

Thus we reduce the problem to the study of the heat kernel transform on $L^{2}\left(\Gamma(\mathbf{l}) \backslash \boldsymbol{H}^{n}\right)$. Using the same techniques employed in [6], we can decompose $L^{2}\left(\Gamma(\mathbf{l}) \backslash \boldsymbol{H}^{n}\right)$ into an orthogonal direct sum of subspaces. This is achieved by means of variants of Weil-Brezin transforms. The image of each of the subspaces under the heat kernel transform is then characterised. As in the case dealt with in [6], we see that twisted Bergman spaces and Hermite-Bergman spaces occur in the characterisation.

This work is closely related to [6] and the proofs are modelled after the corresponding proofs there. Hence more often we omit proofs and supply only those which require a different approach. In the next section, we deal with heat kernel transform on Heisenberg nilmanifolds. The case of H-type nilmanifolds is treated in Section 3.

2000 Mathematics Subject Classification. Primary 22E30; Secondary 35H20, 35K05, 58 J35.

Key words and phrases. H-type groups, representations, Laplacians, heat kernels, Weil-Brezin transforms, Bergman spaces. 
2. Heat kernel transform on Heisenberg nilmanifolds. In this section we consider the heat kernel transform for the Laplacian on a general Heisenberg nilmanifold. Using the structure of such manifolds we reduce the problem to the case of certain special nilmanifolds. We then use variants of the Weil-Brezin transform to study the decomposition of the underlying $L^{2}$ space and obtain its image under the heat kernel transform.

2.1. Heisenberg nilmanifolds. In this subsection we consider Heisenberg nilmanifolds which are defined as quotients of $\boldsymbol{H}^{n}$ by certain lattice subgroups. All such lattices have been characterised and here we recall some results without proof from [14]. Our aim is to look at the right regular representation of $\boldsymbol{H}^{n}$ on $L^{2}\left(\Gamma \backslash \boldsymbol{H}^{n}\right)$ for a general lattice and to obtain a decomposition of $L^{2}\left(\Gamma \backslash \boldsymbol{H}^{n}\right)$ into irreducible subspaces. The main references for this subsection are $[1,2,6,14]$.

A discrete subgroup $\Gamma$ of $\boldsymbol{H}^{n}$ is said to be a lattice subgroup if the quotient $\Gamma \backslash \boldsymbol{H}^{n}$ is compact. The standard example that has been studied in details in [6] is $\Gamma=\boldsymbol{Z}^{n} \times \boldsymbol{Z}^{n} \times$ (1/2) $\boldsymbol{Z}$. The quotient is $\Gamma \backslash \boldsymbol{H}^{n} \simeq \boldsymbol{T}^{2 n} \times \boldsymbol{S}^{1}$ is clearly compact. It is a circle bundle over the torus $\boldsymbol{T}^{2 n}$.

Let $\boldsymbol{A}_{n}$ stand for the group of automorphisms of $\boldsymbol{H}^{n}$ and let $\boldsymbol{A}_{n}^{0}$ be its identity component. This group $\boldsymbol{A}_{n}^{0}$ plays an important role in classifying all the lattices in $\boldsymbol{H}^{n}$. Let $\operatorname{Sp}(2 n, \boldsymbol{R})$ stand for the symplectic group consisting of all $2 n \times 2 n$ matrices preserving the symplectic form $\omega(\xi, \eta)$. That is, $A \in \operatorname{Sp}(2 n, \boldsymbol{R})$ if and only if $\omega(A \xi, A \eta)=\omega(\xi, \eta)$. Every element $A \in \operatorname{Sp}(2 n, \boldsymbol{R})$ defines an automorphism in $\boldsymbol{A}_{n}^{0}$ denoted by the same symbol: $A(\xi, t)=$ $(A \xi, t)$ where $\xi \in \boldsymbol{R}^{2 n}, t \in \boldsymbol{R}$. Note that if $\Gamma$ is a lattice then $A(\Gamma)$ is also a lattice. Thus we have an action of $\boldsymbol{A}_{n}^{0}$ on the set $L\left(\boldsymbol{H}^{n}\right)$ of all lattices in $\boldsymbol{H}^{n}$.

Let $Z$ stand for the centre of $\boldsymbol{H}^{n}$. Observe that $\Gamma \cap Z$ is a nontrivial discrete subgroup of $Z$ and hence there is a unique positive real number $\beta(\Gamma)$ such that

$$
\Gamma \cap Z=\{(0, \beta(\Gamma) m) ; m \in Z\}
$$

The lattice $\pi(\Gamma)$, where $\pi: \boldsymbol{H}^{n} \rightarrow \boldsymbol{R}^{2 n}$ is the projection $\pi(\xi, t)=\xi$, satisfies the condition

$$
[\Gamma, \Gamma] \subset \Gamma \cap Z=\{(0, \beta(\Gamma) m) ; m \in \boldsymbol{Z}\}
$$

Indeed, if $g=(\xi, t)$ and $h=(\eta, s)$ then $[g, h]=(0, \omega(\xi, \eta))$. Actually we have $\omega(\pi(\Gamma), \pi(\Gamma))=\beta(\Gamma) Z$ whenever $\Gamma$ is a lattice in $\boldsymbol{H}^{n}$. This motivates us to make the following definition.

A lattice $D$ in $\boldsymbol{R}^{2 n}$ is said to be a Heisenberg lattice if $\omega(D, D)=l Z$ for some $l>0$. The collection of such lattices will be denoted by $H L\left(\boldsymbol{R}^{2 n}\right)$. Thus $\pi(\Gamma) \in H L\left(\boldsymbol{R}^{2 n}\right)$ for any lattice $\Gamma$ in $\boldsymbol{H}^{n}$ and vice versa. We recall the following result of Tolimieri (see [14, Corollary after Lemma 1.8]).

THEOREM 2.1. There is one to one correspondence between lattices in $\boldsymbol{H}^{n}$ and Heisenberg lattices in $\boldsymbol{R}^{2 n}$. 
To each Heisenberg lattice $D$, one can associate $n$ positive real numbers $l_{1}, l_{2}, \ldots, l_{n}$ with the property that $l_{j}$ divides $l_{j+1}$. Set

$$
\boldsymbol{Z}^{n *}=\left\{\mathbf{I}=\left(l_{1}, l_{2}, \ldots, l_{n}\right) ; l_{j+1} l_{j}^{-1} \in \mathbf{Z}\right\} .
$$

Let $e_{j}, 1 \leq j \leq 2 n$ be the standard coordinate vectors in $\boldsymbol{R}^{2 n}$. For $\mathbf{l} \in \boldsymbol{Z}^{n *}$ denote $D(\mathbf{l})=$ $\left[e_{1}, e_{2}, \ldots, e_{n}, l_{1} e_{n+1}, l_{2} e_{n+2}, \ldots, l_{n} e_{2 n}\right]$ be the $\boldsymbol{Z}$-module of $\boldsymbol{R}^{2 n}$ spanned by the vectors $e_{1}, e_{2}, \ldots, e_{n}, l_{1} e_{n+1}, \ldots, l_{n} e_{2 n}$. Then it is clear that $D(\mathbf{l}) \in H L\left(\boldsymbol{R}^{2 n}\right)$ and $\omega(D(\mathbf{l}), D(\mathbf{l}))=$ Z.

THEOREM 2.2 ([14, Theorem 1.9]). For each $D \in H L\left(\boldsymbol{R}^{2 n}\right)$ there exist a unique $\mathbf{l} \in$ $Z^{n *}$, a unique $d>0$ and an $A \in \operatorname{Sp}(2 n, \boldsymbol{R})$ such that $D=A(d . D(\mathbf{I}))$.

Now combining the above theorems we can obtain the following result which gives the structure of all lattices in $\boldsymbol{H}^{n}$. Given $\mathbf{l} \in \boldsymbol{Z}^{n *}$, let $\Gamma$ (l) be the subgroup of $\boldsymbol{H}^{n}$ generated by

$$
\widetilde{e_{1}}, \widetilde{e_{2}}, \ldots, \widetilde{e_{2 n}} \text {, }
$$

where $\widetilde{e_{i}}=\left(e_{i}, 0\right)$ for $i=1,2,3, \ldots, n$ and $\widetilde{e_{n+j}}=\left(l_{j} e_{n+j}, 0\right)$ for $j=1,2, \ldots, n$. Then we have the following result from Tolimieri [14]. We denote the collection of all lattices on the Heisenberg group by $L\left(\boldsymbol{H}^{n}\right)$.

THEOREM 2.3 ([14, Theorem 1.10]). For each $\Gamma \in L\left(\boldsymbol{H}^{n}\right)$ there exist a unique $\mathbf{l} \in$ $Z^{n *}$, a unique $d>0$ and an $A \in A_{n}$ such that $\Gamma=A(d . \Gamma(\mathbf{l}))$.

In view of the above theorem, in studying the heat kernel transform we can restrict ourselves to lattices of the form $\Gamma(\mathbf{I})$.

2.2. Analysis on the nilmanifold $\Gamma(\mathbf{I}) \backslash \boldsymbol{H}^{n}$. In this subsection we consider the lattice $\Lambda=\Gamma(\mathbf{l})$, defined in the previous subsection, and consider the nilmanifold $M=\Gamma(\mathbf{I}) \backslash \boldsymbol{H}^{n}$ associated to it. As we remarked earlier, there is no loss of generality in doing so. The Lebesgue measure on $\boldsymbol{H}^{n}$ induces an invariant measure on $M$. So we get a unitary representation $R$ of $\boldsymbol{H}^{n}$ on $L^{2}(M)$ defined by

$$
R(g) F(\Lambda h)=F(\Lambda h g), \quad F \in L^{2}(M), \quad g, h \in \boldsymbol{H}^{n} .
$$

We can identify functions on $M$ with functions on $\boldsymbol{H}^{n}$ that are invariant under left translations by elements of $\Lambda$. Since $\Lambda=\operatorname{span}\left\{\widetilde{e_{1}}, \widetilde{e_{2}}, \ldots, \widetilde{e_{2 n}}\right\}$, it is clear that

$$
\Lambda=\boldsymbol{Z}^{n} \times l_{1} \boldsymbol{Z} \times l_{2} \boldsymbol{Z} \times \cdots \times l_{n} \boldsymbol{Z} \times\left(l_{1} / 2\right) \boldsymbol{Z}
$$

and any element $h \in \Lambda$ will be $h=\left(p, q_{1} l_{1}, q_{2} l_{2}, \ldots, q_{n} l_{n},\left(l_{1} / 2\right) r\right)$, where $p, q_{1}, \ldots, q_{n}, r$ are integers. To make the notation simple we denote $\left(q_{1} l_{1}, q_{2} l_{2}, \ldots, q_{n} l_{n}\right)$ by $q l$. So we can write any element $h \in \Lambda$ as $h=\left(p, q l,\left(l_{1} / 2\right) r\right)$.

Therefore, every $\Lambda$-invariant function is $\left(l_{1} / 2\right)$-periodic in the central variable. Thus by defining

$$
H_{k}(\Lambda)=\left\{F \in L^{2}(M) ; F(\xi, t)=e^{4 \pi i k t / l_{1}} F(\xi, 0)\right\}
$$


we get the orthogonal direct sum decomposition

$$
L^{2}(M)=\sum_{k \in \boldsymbol{Z}} H_{k}(\Lambda) .
$$

Recall that for each $\lambda \in \boldsymbol{R}, \lambda \neq 0$, the Schrödinger representation $\pi_{\lambda}$ of $\boldsymbol{H}^{n}$ is given by,

$$
\pi_{\lambda}(x, u, \xi) \phi(v)=e^{i \lambda \xi} e^{i \lambda(x \cdot v+(1 / 2) x \cdot u)} \phi(v+u) .
$$

It is easy to check that each $H_{k}(\Lambda)$ is $R$-invariant, and hence for every $k \neq 0$ the Stone-von Neumann theorem says that the restriction of $R$ to $H_{k}(\Lambda)$ decomposes into a direct sum of irreducible representations each of which is unitarily equivalent to $\pi_{4 \pi k} / l_{1}$.

Each of the $H_{k}(\Lambda)$ can be further decomposed into orthogonal subspaces each of which will be the image of $L^{2}\left(\boldsymbol{R}^{n}\right)$ under a unitary operator. For the standard lattice $\Gamma(1,1, \ldots, 1)$, such a decomposition has been obtained in [6] (see also [12]). For the general case we refer to $[2,6,14]$. As we closely follow [12] in obtaining this decomposition, we will be only sketchy in our proof.

We first consider Weil-Brezin transform $V_{k}$ defined on the Schwartz class $\mathcal{S}\left(\boldsymbol{R}^{n}\right)$ by

$$
V_{k} f(x, u, \xi)=e^{i \lambda \xi} e^{i(\lambda / 2) x \cdot u} \sum_{m \in Z^{n}} e^{i \lambda \sum_{j=1}^{n} l_{j} m_{j} x_{j}} f(u+m l),
$$

where $m l=\left(m_{1} l_{1}, m_{2} l_{2}, \ldots, m_{n} l_{n}\right)$. It is easy to check that $V_{k} f$ is $\Lambda$-invariant. Further it can be shown that the $L^{2}(M)$ norm of $V_{k} f$ is just $\|f\|_{2}$ and hence $V_{k}$ can be extended to the whole of $L^{2}\left(\boldsymbol{R}^{n}\right)$ as an isometry onto $H_{k}(\Lambda)$. We would like to decompose $H_{k}(\Lambda)$ further into mutually orthogonal subspaces.

To effect this decomposition, we introduce the finite group $\boldsymbol{A}_{k}$ which is defined by

$$
\boldsymbol{A}_{k}=\boldsymbol{Z} / 2 k \boldsymbol{Z} \times \boldsymbol{Z} / 2 k p_{2} \boldsymbol{Z} \times \cdots \times \boldsymbol{Z} / 2 k p_{n} \boldsymbol{Z},
$$

where $p_{i}=l_{i} / l_{1}$ for $i=1,2,3, \ldots, n$. For each $j \in \boldsymbol{A}_{k}$ define

$$
V_{k, j} f(x, u, \xi)=e^{2 \pi i j \cdot x} V_{k} f(x, u, \xi) .
$$

Let $H_{k, j}$ be the image of $L^{2}\left(\boldsymbol{R}^{n}\right)$ under $V_{k, j}$. Then we have the following decomposition.

Proposition 2.4. For each $k, H_{k}$ is the orthogonal direct sum of the spaces $H_{k, j}$, $j \in \boldsymbol{A}_{k}$.

The proof of this proposition depends on several results. The orthogonality can be proved by a direct decomposition. In what follows, we only indicate how a function $F \in H_{k}$ can be decomposed into a sum of elements of $H_{k, j}$.

Defining $G(x, u)=F(x, u, 0)$ we see that the $\Lambda$-invariance of $F$ translates into the condition

$$
G(x+m, u+n l)=e^{i(\lambda / 2)(m \cdot u-x \cdot n l)} G(x, u) .
$$

We will show that every $G(x, u)$ satisfying the above condition can be further decomposed as $G(x, u)=\sum_{j \in A_{k}} G_{j}$, where $G_{j}$ satisfies some extra conditions. 
To this end, we define $G_{j, m}(x, u)$ to be

$$
e^{-\pi i \sum_{j=1}^{n}\left(m_{j} / l_{j}\right) u_{j}} e^{-\pi(i / k) \sum_{i=1}^{n}\left(m_{i} / p_{i}\right) j_{i}} G\left(x_{1}+\frac{1}{2 k} m_{1}, \ldots, x_{n}+\frac{1}{2 k p_{n}} m_{n}, u\right)
$$

and consider the sum $\sum_{j \in A_{k}} \sum_{m \in A_{k}} G_{j, m}(x, u)$ which is given by

$$
\sum_{m \in A_{k}} e^{-\pi i \sum_{j=1}^{n}\left(m_{j} / l_{j}\right) u_{j}} G\left(x_{1}+\frac{1}{2 k} m_{1}, \ldots, x_{n}+\frac{1}{2 k p_{n}} m_{n}, u\right) I_{k}(m),
$$

where

$$
I_{k}(m)=\left(\sum_{j \in \boldsymbol{A}_{k}} e^{-\pi(i / k) \sum_{i=1}^{n}\left(m_{i} / p_{i}\right) j_{i}}\right)
$$

Observe that we have

$$
\sum_{j \in \boldsymbol{A}_{k}} e^{-\pi(i / k) \sum_{i=1}^{n}\left(m_{i} / p_{i}\right) j_{i}}=0
$$

unless $m=0$ in which case the sum equals $(2 k) \times \cdots \times\left(2 k p_{n}\right)$. Consequently, we have

$$
\sum_{j \in A_{k}} \sum_{m \in A_{k}} G_{j, m}(x, u)=\prod_{i=1}^{n}\left(2 k p_{i}\right) G(x, u) .
$$

Therefore, by defining

$$
G_{j}(x, u)=\prod_{i=1}^{n}\left(2 k p_{i}\right)^{-1} \sum_{m \in A_{k}} G_{j, m}(x, u),
$$

we get the decomposition $G=\sum_{j \in A_{k}} G_{j}$.

We now claim that $G_{j}$ satisfies the extra condition

$$
G_{j}\left(x_{1}+\frac{1}{2 k} d_{1}, \ldots, x_{n}+\frac{1}{2 k p_{n}} d_{n}, u\right)=e^{\pi i(d / l) \cdot u} e^{\pi i(1 / k)(d / p) \cdot j} G_{j}(x, u) .
$$

To see this, we first observe that

$$
\begin{aligned}
& G_{j}\left(x_{1}+\frac{1}{2 k} d_{1}, \ldots, x_{n}+\frac{1}{2 k p_{n}} d_{n}, u\right) \\
& \quad=e^{\pi i(d / l) \cdot u} e^{\pi i(1 / k)(d / p) \cdot j} \sum_{m \in A_{k}} G_{j, m+d}(x, u) .
\end{aligned}
$$

From the definition of $G_{j}$ it follows, using the quasi-periodicity of $G$, that

$$
\sum_{m \in A_{k}} G_{j, m}(x, u)=\sum_{m \in A_{k}} G_{j, m+d}(x, u),
$$

and hence the claim is proved.

In order to complete the proof of the proposition we need to show that each $F_{j}(x, u, \xi)$ is equal to $e^{i \lambda(k) \xi} G_{j}(x, u)$, where $\lambda(k)=4 \pi k / l_{1}$ can be written as $F_{j}=V_{k, j} f_{j}$ for some $f_{j} \in$ $L^{2}\left(\boldsymbol{R}^{n}\right)$. In order to prove this we need the following two propositions. The first proposition 
deals with tempered distributions $v$ on $\boldsymbol{R}^{n}$ that are invariant under $\rho_{k}(\Lambda)$ for $\rho_{k}=\pi_{\lambda(k)}$. If $v$ is such a ditribution then $F(x, u, \xi)=\left(v, \pi_{\lambda}(x, u, \xi) f\right)$ where $f$ is a Schwartz function on $\boldsymbol{R}^{n}$, gives a $\Lambda$-invariant function on $\boldsymbol{H}^{n}$. In view of this, the following proposition plays an important role in the decomposition of $H_{k}$.

PROPOSITION 2.5. Every tempered distribution $v$ invariant under $\rho_{k}(\Lambda)$ is of the form $v=\sum_{j \in A_{k}} c_{j} v_{j}$ with $v_{j}$ defined by

$$
\left(v_{j}, f\right)=\sum_{m \in Z^{n}} \hat{f}\left(\frac{j_{1}+2 k m_{1}}{l_{1}}, \frac{j_{2}+2 k m_{2} p_{2}}{l_{2}}, \ldots, \frac{j_{n}+2 k m_{n} p_{n}}{l_{n}}\right) .
$$

Here $\hat{f}$ denotes the Fourier transform of the Schwartz class function $f$.

The proof of the above proposition is very similar to that of [6, Proposition 3.1] except for some technicalities, so we skip it here.

We can also show that the matrix coefficients $\left(v_{j}, f\right)$ can be expressed in terms of the Weil-Brezin transforms $V_{k, j}$.

Proposition 2.6. For each $f \in \mathcal{S}\left(\boldsymbol{R}^{n}\right)$, we have

$$
F_{j}(x, u, \xi)=V_{k, j} g_{j}\left(\frac{u}{l},-l x, \xi\right)
$$

where $u / l=\left(u_{1} / l_{1}, \ldots, u_{n} / l_{n}\right), l x=\left(l_{1} x_{1}, l_{2} x_{2}, \ldots, l_{n} x_{n}\right)$ and $f$ and $g_{j}$ are related by

$$
g_{j}\left(l_{1} s_{1}, \ldots, l_{n} s_{n}\right)=\hat{f}\left(\frac{j_{1}}{l_{1}}+\frac{2 k}{l_{1}} s_{1}, \ldots, \frac{j_{n}}{l_{n}}+\frac{2 k}{l_{1}} s_{n}\right) .
$$

For a proof of this proposition in the case of the standard lattice, we refer to [6]. The same proof can be modified to suit the present case. Making use of the above two propositions we can now complete the proof of Proposition 2.4. Again the proof is similar to that of the standard lattice case. We provide a proof just for the sake of completeness.

It only remains to show that $G_{j}$ can be written as

$$
G_{j}(x, u)=V_{k, j} f_{j}(x, u, 0), f_{j} \in L^{2}\left(\boldsymbol{R}^{n}\right) .
$$

To prove this, we consider

$$
g_{j}(x, u)=e^{-2 \pi i j \cdot x} e^{-i(\lambda / 2) x \cdot u} G_{j}(x, u),
$$

where $\lambda=4 \pi k / l_{1}, x=\left(x_{1}, x_{2}, \ldots, x_{n}\right)$. It is clear that the function $g_{j}$ is $\left(1 / 2 k, 1 / 2 k p_{2}, \ldots\right.$, $1 / 2 k p_{n}$ )-periodic in the variables $x$. Therefore, it admits an expansion of the form

$$
g_{j}(x, u)=\sum_{m \in Z^{n}} c_{m}(u) e^{i \lambda m l \cdot x},
$$

where $m l=\left(m_{1} l_{1}, m_{2} l_{2}, \ldots, m_{n} l_{n}\right)$ and the Fourier coefficients are given by

$$
c_{m}(u)=\int_{[0,1 / 2 k)} \cdots \int_{\left[0,1 / 2 k p_{n}\right)} g_{j}(x, u) e^{-i \lambda m l \cdot x} d x .
$$


Now the transformation property of $G_{j}(x, u)$ leads to

$$
g_{j}(x, u-m l)=e^{i \lambda m l \cdot x} g_{j}(x, u) .
$$

From this relation it is then obvious that $c_{m}(u)=c_{0}(u+m l)$. Hence we have

$$
G_{j}=e^{2 \pi i j \cdot x} e^{i(\lambda / 2) x \cdot u} \sum_{m \in Z^{n}} c_{0}(u+m l) e^{i \lambda m l \cdot x}=V_{k, j} f_{j}(x, u, 0),
$$

where $f_{j}=c_{0}$ and $c_{0} \in L^{2}\left(\boldsymbol{R}^{n}\right)$.

2.3. Heat kernel transform on $\Gamma(\mathbf{l}) \backslash \boldsymbol{H}^{n}$. In this subsection we consider the image of $L^{2}\left(\Gamma(\mathbf{I}) \backslash \boldsymbol{H}^{n}\right)$ under the heat kernel transform. Let $\Delta$ stand for the standard left invariant Laplacian on $\boldsymbol{H}^{n}$ and let $k_{t}$ be the associated heat kernel. It is explicitly given by

$$
k_{t}(x, u, \xi)=c_{n} \int_{-\infty}^{\infty}\left(\frac{\lambda}{\sinh t \lambda}\right)^{n} e^{-t \lambda^{2}} e^{i \lambda \xi} e^{-(\lambda / 4)(\operatorname{coth} t \lambda)\left(x^{2}+u^{2}\right)} d \lambda .
$$

The heat semigroup is defined by $S_{t} f=f * k_{t}$, and for $f \in L^{2}\left(\boldsymbol{H}^{n}\right)$ it can be shown that $S_{t} f$ extends to $\boldsymbol{C}^{2 n+1}$ as an entire function. This transform taking $f$ into the entire function $S_{t} f$ is called the heat kernel transform.

The image of $L^{2}\left(\boldsymbol{H}^{n}\right)$ under the heat kernel transform has been studied in [5]. One can also restrict the heat kernel transform to $L^{2}(M)$ where $M$ is a nilmanifold and ask for a characterisation of the image. When $M$ is the nilmanifold associated to the standard lattice, this has been done in [6]. Here we take up the general case. In view of the structure of Heisenberg lattices, it is enough to look at the case $\Lambda=\Gamma(\mathbf{l})$. Let $S_{t}^{\Lambda}$ stand for the heat kernel transform restricted to $L^{2}\left(\Lambda \backslash \boldsymbol{H}^{n}\right)$. It is easy to see that $S_{t}^{\Lambda}$ leaves each of $H_{k}$ invariant, and hence it is enough to characterise $S_{t}^{\Lambda}\left(H_{k}\right)$ for each $k \geq 0$. We assume $k \neq 0$ as the case $k=0$ can be handled as in [6].

In order to describe $S_{t}^{\Lambda}\left(H_{k}\right)$ we define certain spaces of entire functions. Let $\Upsilon=Z^{n} \times$ $\left(l_{1} \boldsymbol{Z} \times l_{2} \boldsymbol{Z} \times \cdots \times l_{n} \boldsymbol{Z}\right)$. We let $\mathcal{H}_{k}\left(\boldsymbol{R}^{2 n}, \Upsilon\right)$ stand for the space of all functions $F(x, u)$ for which $e^{i \lambda \xi} F(x, u) \in H_{k}$. These functions are characterised by the property

$$
F(x+m, u+n l)=e^{i \lambda(u \cdot m-x \cdot n l)} F(x, u)
$$

for $(m, n l) \in \Upsilon$. Then for $k \neq 0$ we define $\mathcal{H}_{k}^{t}\left(C^{2 n}, \Upsilon\right)$ to be the space of all functions in $\mathcal{H}_{k}\left(\boldsymbol{R}^{2 n}, \Upsilon\right)$ having entire extension to $\boldsymbol{C}^{2 n}$ and satisfying

$$
\|F\|_{k, t}^{2}=\int_{\boldsymbol{R}^{2 n}}\left(\int_{Q(l)} \int_{Q}|F(z, w)|^{2} W_{t}^{\lambda(k)}(z, w) d u d x\right) d y d v<\infty,
$$

where $Q(l)=\left[0, l_{1}\right) \times \cdots \times\left[0, l_{n}\right), Q=[0,1)^{n}$ and

$$
W_{t}^{\lambda}(k)(z, w)=2^{n} e^{i \lambda(k)(u \cdot y-v \cdot x)} e^{-\lambda(k) \operatorname{coth}(2 t \lambda(k))\left(y^{2}+v^{2}\right)} .
$$

For each $j \in \boldsymbol{A}_{k}$ we also define the spaces $\mathcal{H}_{k, j}^{t}\left(\boldsymbol{C}^{2 n}, \Upsilon\right)$ as the subspaces of $\mathcal{H}_{k}^{t}\left(\boldsymbol{C}^{2 n}, \Upsilon\right)$ satisfying the extra condition

$$
G\left(z_{1}+\frac{1}{2 k} d_{1}, \ldots, z_{n}+\frac{1}{2 k p_{n}} d_{n}, w\right)=e^{\pi i(d / l) \cdot u} e^{\pi(i / k)(d / p) \cdot j} G(z, w),
$$


where $(d / p) \cdot j=\sum_{i=1}^{n}\left(d_{i} / p_{i}\right) j_{i}$ and $(d / l) \cdot u=\sum_{i=1}^{n}\left(d_{i} / l_{i}\right) u_{i}$. Then we have the following proposition.

PROPOSITION 2.7. $\mathcal{H}_{k}^{t}\left(\boldsymbol{C}^{2 n}, \Upsilon\right)$ is the orthogonal direct sum of $\mathcal{H}_{k, j}^{t}\left(\boldsymbol{C}^{2 n}, \Upsilon\right)$ as $j$ varies over $\boldsymbol{A}_{k}$.

For a proof of this proposition and also for the proof of the following, we refer to [6]. The required modifications are left to the reader. The relation between $\mathcal{H}_{k, j}^{t}\left(\boldsymbol{C}^{2 n}, \Upsilon\right)$ and $S_{t}^{\Lambda}$ is as follows.

THEOREM 2.8. An entire function $F(z, w)$ belongs to $\mathcal{H}_{k, j}^{t}\left(\boldsymbol{C}^{2 n}, \Upsilon\right)$ if and only if $F(z, w)=e^{t \lambda^{2}} V_{k, j} f * k_{t}(z, w, 0)$ for some $f \in L^{2}\left(\boldsymbol{R}^{n}\right)$.

The proof of this theorem given in [6] for the standard lattice case makes use of HermiteBergman spaces. The Weil-Brezin transforms can be defined on these Hermite-Bergman spaces and they intertwine the heat kernel transform. We refer to [6, Proposition 4.6]. Finally the image of $L^{2}\left(\Lambda \backslash \boldsymbol{H}^{n}\right)$ under $S_{t}^{\Lambda}$ can be described as follows.

THEOREM 2.9. The image of $L^{2}\left(\Lambda \backslash \boldsymbol{H}^{n}\right)$ under $S_{t}^{\Lambda}$ is the direct sum of $e^{i \lambda(k) \zeta} \mathcal{H}_{k, j}^{t}\left(\boldsymbol{C}^{2 n}, \Upsilon\right), k \in \boldsymbol{Z}, j \in \boldsymbol{A}_{k}$; that is,

$$
S_{t}^{\Lambda}\left(L^{2}\left(\Lambda \backslash \boldsymbol{H}^{n}\right)\right)=\sum_{k=-\infty}^{\infty} \sum_{j \in \boldsymbol{A}_{k}} e^{2 t \lambda(k)^{2}} e^{i \lambda(k) \zeta} \mathcal{H}_{k, j}^{t}\left(\boldsymbol{C}^{2 n}, \Upsilon\right) .
$$

REMARK 2.1. Now if $\Lambda=A(d . \Gamma(1))$ then $\beta(\Lambda)=d^{2}$ and so $\lambda=4 \pi k / d^{2}$. Then for any $\Lambda$-invariant function $F, d^{-1} A^{-1} \circ F$ is a $\Gamma(1)$-invariant function. So then using $V_{k, j}$, the corresponding $V_{k, j, \Lambda}$ can be defined as $V_{k, j, \Lambda}=d^{-1} A^{-1} \circ V_{k, j}$ and hence the same type of results can be deduced.

3. Heat kernel transform on H-type nilmanifolds. In this section we study the heat kernel transform on H-type groups $N$ and their nilmanifolds. Using a partial Radon transform we reduce the problem on $N$ to a problem on $\boldsymbol{H}^{n}$. The problem on H-type nilmanifolds is also reduced to the case of Heisenberg nilmanifolds.

3.1. H-type groups. H-type Lie algebras and Lie groups were introduced by Kaplan [4]. We say that a Lie algebra $\mathfrak{n}$ is of H-type if it is the direct sum $\mathfrak{v} \oplus \mathfrak{z}$ of two Euclidean spaces with a Lie algebra structure such that $\mathfrak{z}$ is the center of $\mathfrak{n}$ and for all unit vector $v \in \mathfrak{v}$ the map $a d(v)$ is a surjective isometry of the orthogonal complement of kerad $(v)$ onto $\mathfrak{z}$. For such an algebra we define a map $J: \mathfrak{z} \rightarrow \operatorname{End}(\mathfrak{v})$ by

$$
\left(J_{\omega} v, v^{\prime}\right)=\left(\omega,\left[v, v^{\prime}\right]\right), \quad \omega \in \mathfrak{z}, v, v^{\prime} \in \mathfrak{v} .
$$

It then follows that $J_{\omega}^{2}=-I$ whenever $\omega$ is a unit vector and hence $J_{\omega}$ defines a complex structure on $\mathfrak{v}$. The Hermitian inner product corresponding to this complex structure is given by

$$
\langle(v, w\rangle)_{\omega}=(v, w)+i\left(J_{\omega} v, w\right)=(v, w)+i([v, w], \omega) .
$$


Let $2 n$ and $m$ be the dimensions of $\mathfrak{v}$ and $\mathfrak{z}$, respectively.

A step two nilpotent Lie group $N$ is said to be an H-type group if its Lie algebra $\mathfrak{n}$ is of H-type. Identifying the group with its Lie algebra, we write the elements of $N$ as $(v, z)$ and, in view of the Baker-Campbell- Hausdorff formula, the group law takes the form

$$
(v, z)\left(v^{\prime}, z^{\prime}\right)=(v, z)+\left(v^{\prime}, z^{\prime}\right)+\frac{1}{2}\left[(v, z),\left(v^{\prime}, z^{\prime}\right)\right] .
$$

The Heisenberg group $\boldsymbol{H}^{n}$ is an H-type group. To every H-type algebra, we can associate Heisenberg algebras as follows. Given a unit vector $\omega$ in $\mathfrak{z}$, let $k(\omega)$ stand for its orthogonal complement in $\mathfrak{z}$. Then the quotient algebra $\mathfrak{n}(\omega)=\mathfrak{n} / k(\omega)$ can be identified with $\mathfrak{v} \oplus \boldsymbol{R}$ by setting

$$
\left[(v, t),\left(v^{\prime}, t^{\prime}\right)\right]_{\omega}=\left(0,\left(J_{\omega} v, v^{\prime}\right)\right) .
$$

It has been shown in Ricci [11] that this algebra is isomorphic to the Heisenberg algebra $\mathfrak{h}^{n}$. We denote this group by $\boldsymbol{H}_{\omega}^{n}$.

The above connection with Heisenberg algebras makes simple the representation theory of H-type groups. The irreducible unitary representations of $N$ comes in two groups. The one-dimensional representations do not occur in the Plancherel formula and hence we do not consider them. If $\pi$ is any infinite-dimensional irreducible unitary representation, then its restriction to the center is a character and hence $\pi(0, z)=e^{i \lambda(\omega, z)}$ Id for some $\lambda>0$ and $\omega \in$ $S^{m-1}$, where $m$ is the dimension of $\mathfrak{z}$. The representation $\pi$ factors through a representation of $\boldsymbol{H}_{\omega}^{n}$. By making use of the Stone-von Neumann theorem we can show that all irreducible unitary representations are parametrised by $(\lambda, \omega)$. We denote such a representation by $\pi_{\lambda, \omega}$.

The Plancherel theorem for $N$ can be deduced from that of $\boldsymbol{H}^{n}$ by making use of (partial) Radon transform. As we need to use this, we briefly recall the definition and some properties. Given an integrable function $f$ on $N$ and $\omega \in S^{m-1}$, we define a function on $\boldsymbol{H}_{\omega}^{n}$ by

$$
f_{\omega}(v, s)=\int_{k(\omega)} f(v, s \omega+\eta) d \eta
$$

The collection $f_{\omega}$ completely determines $f$. Moreover, it can be verified that

$$
(f * g)_{\omega}(v, s)=f_{\omega} * g_{\omega}(v, s),
$$

where the first convolution is in $N$ and the second in $\boldsymbol{H}_{\omega}^{n}$. We also remark that $\pi_{\lambda, \omega}(f)=$ $\pi_{\lambda}\left(f_{\omega}\right)$ where $\pi_{\lambda}$ is the Schrödinger representation of $\boldsymbol{H}_{\omega}^{n}$.

3.2. Heat kernel transform on $\mathbf{H}$-type groups. We fix an orthonormal basis $X_{j}, j=$ $1,2, \ldots, 2 n$, for the Lie algebra $\mathfrak{v}$ and define the sublaplacian $\mathcal{L}=-\sum_{j=1}^{2 n} X_{j}^{2}$ as in the case of the Heisenberg groups. Then it is known that $\mathcal{L}$ generates a diffusion semigroup which is given by a kernel $p_{t}$. This kernel has been explicitly calculated by Cygan [3] and Randall [10]. Indeed, we have

$$
p_{t}(v, z)=c_{n} \int_{\boldsymbol{R}^{m}} e^{-i u \cdot z}\left(\frac{|u|}{\sinh t|u|}\right)^{n} e^{-(1 / 4)|u|(\operatorname{coth} t|u|)|v|^{2}} d u .
$$

This kernel is a positive Schwartz class function for which good estimates can be proved. 
Let $Z_{j}=\partial / \partial z_{j}, j=1,2, \ldots, m$, and consider the full Laplacian $\Delta=\mathcal{L}-\sum_{j=1}^{m} Z_{j}^{2}$. The heat kernel associated to this operator is given by

$$
q_{t}(v, z)=c_{n} \int_{\boldsymbol{R}^{m}} e^{-t|u|^{2}} e^{-i u \cdot z}\left(\frac{|u|}{\sinh t|u|}\right)^{n} e^{-(1 / 4)|u|(\operatorname{coth} t|u|)|v|^{2}} d u .
$$

It then follows that $q_{t}(v, z)$ can be holomorphically extended to $\boldsymbol{C}^{2 n} \times \boldsymbol{C}^{m}$ as an entire function. For $f \in L^{2}(N)$ the function $f * q_{t}(v, z)$, which solves the heat equation for $\Delta$, also extends to $\boldsymbol{C}^{2 n} \times \boldsymbol{C}^{m}$ as an entire function. We are interested in this transform taking $f$ into the holomorphically extendable function $f * q_{t}$. We can ask for a characterisation of the image of $L^{2}(N)$ under this transform.

In [5], this problem was treated for the Heisenberg group and it was shown that the image is not a weighted Bergman transform in sharp contrast to the Euclidean case. There the authors have obtained different characterisations. In this section we state and prove one such characterisation for the heat kernel transform on $N$. For the motivation of the following we refer to [13].

Consider the representations $\pi_{\lambda, \omega}$ realised on a Hilbert space $\mathcal{H}$. Given $f \in L^{2}(N)$ the operator $\pi_{\lambda, \omega}(f)$ is Hilbert-Schmidt. The representations $\pi_{\lambda, \omega}$ can also be realised on the space of Hilbert-Schmidt operators on $\mathcal{H}$ simply defining $\pi_{\lambda, \omega}(x, u, \xi) T$ as the action on a Hilbert-Schmidt operator $T$. Note that we have slightly changed our notation and written $(x, u, \xi), x, u \in \boldsymbol{R}^{n}, \xi \in \boldsymbol{R}^{m}$, for the elements of $N$. We can therefore, consider the operator valued function $(x, u, \xi) \mapsto \pi_{\lambda, \omega}(x, u, \xi) \pi_{\lambda, \omega}(F)$ and ask if it can be holomorphically extended to $\boldsymbol{C}^{n} \times \boldsymbol{C}^{n} \times \boldsymbol{C}^{m}$. We can show that it is so precisely when $F=f * q_{t}$ for some $f \in L^{2}(N)$. Thus we get the following characterisation of the image of $L^{2}(N)$ under the heat kernel transform.

THEOREM 3.1. A function $F$ belongs to the image of $L^{2}(N)$ under the heat kernel transform if and only if $(x, u, \xi) \mapsto \pi_{\lambda, \omega}(x, u, \xi) \pi_{\lambda, \omega}(F)^{*}$ extends to $\boldsymbol{C}^{n} \times \boldsymbol{C}^{n} \times \boldsymbol{C}^{m}$ as an entire function so that

$$
\left\|\pi_{\lambda, \omega}(i(y, v, \eta)) \pi_{\lambda, \omega}(F)^{*}\right\|_{H S}
$$

is square integrable over $N \times \boldsymbol{R}^{m}$ with respect to the measure

$$
e^{-(1 / 2 t)|\eta|^{2}} p_{2 t}^{\lambda}(2 y, 2 v)|\lambda|^{n+m-1} d(y, v, \eta) d \lambda d \omega .
$$

If $F=f * q_{t}$ the above integral is a constant multiple of $\|f\|_{2}^{2}$.

PROOF. Using partial Radon transform, we can quickly reduce the theorem to the case of the Heisenberg group. Indeed, as we have already remarked, $\pi_{\lambda, \omega}(F)=\pi_{\lambda}\left(F_{\omega}\right)$. We also know that $\left(q_{t}\right)_{\omega}=k_{t}$, the heat kernel on $\boldsymbol{H}_{\omega}^{n}$. Therefore, if $F=f * q_{t}$ then $\pi_{\lambda, \omega}(F)=$ $\pi_{\lambda}\left(f_{\omega} * k_{t}\right)$. The function $f_{\omega}$ does not belong to $L^{2}\left(\boldsymbol{H}_{\omega}^{n}\right)$ but the modified Radon transform

$$
R_{\omega} f(x, u, s)=D_{s}^{(m-1) / 2} f_{\omega}(x, u, s),
$$


where $D_{s}^{(m-1) / 2}$ is the fractional derivative of order $(m-1) / 2$, does. Since $\pi_{\lambda}\left(R_{\omega} f\right)=$ $|\lambda|^{(m-1) / 2} \pi_{\lambda}\left(f_{\omega}\right)$, we can write

$$
\pi_{\lambda, \omega}(x, u, \xi) \pi_{\lambda, \omega}\left(f * q_{t}\right)^{*}=|\lambda|^{(1-m) / 2} e^{i \lambda \omega \cdot \xi} \pi_{\lambda}(x, u, 0) \pi_{\lambda}\left(R_{\omega} f * k_{t}\right)^{*} .
$$

We can therefore appeal to [13, Theorem 13.5] to conclude that

$$
(x, u, \xi) \mapsto \pi_{\lambda, \omega}(x, u, \xi) \pi_{\lambda, \omega}(F)^{*}
$$

extends to $\boldsymbol{C}^{n} \times \boldsymbol{C}^{n} \times \boldsymbol{C}^{m}$ as an entire function with the stated integrability condition.

By the same result, we can also conclude that the integral mentioned in the statement of the theorem reduces to

$$
\int_{S^{m-1}}\left(\int_{\boldsymbol{R}^{2 n+1}}\left|R_{\omega} f(x, u, s)\right|^{2} d(x, u, s)\right) d \omega,
$$

which is nothing but $\|f\|_{2}^{2}$. This proves half of the theorem. The other half can be proved, again using [13, Theorem 13.5] and the inversion formula for the modified Radon transform.

3.3. Nilmanifolds associated to H-type groups. We consider a nilmanifold $M=$ $\Gamma \backslash N$ where $N$ is an H-type group and $\Gamma$ is a lattice subgroup such that $M$ is compact. Such subgroups $\Gamma$ are characterised by the property that $\log \Gamma$ is a subgroup of the underlying additive group of the Lie algebra $\mathfrak{n}$. Here log stands for the inverse of the expenential map exp:n $\rightarrow N$. Given the nilmanifold $\Gamma \backslash N$, we form $L^{2}(\Gamma \backslash N)$ using an $N$-invariant measure. We can then define the right regular representation $U_{\Gamma}$ of $N$ on $L^{2}(\Gamma \backslash N)$. We are interested in decomposing $L^{2}(\Gamma \backslash N)$ into subspaces that are irreducible under the action of $U_{\Gamma}$.

This problem has been addressed in the more general context of nilmanifolds in Brezin [2] and Auslander-Brezin [1]. For step two nilpotent Lie groups, Brezin [2] has reduced the problem to the case of Heisenberg groups. For Heisenberg nilmanifolds he has used variants of the Weil construction (which we have already seen in Section 2) to get the decompostion. In the general case he has produced an algorithm which enables one to reduce the general case to the step two case. The decomposition obtained by Brezin in the step two case is not good enough for our purpose of studying heat kernel transforms. As we are dealing with a special class of step two groups, we can obtain a very explicit decomposition of $L^{2}(\Gamma \backslash N)$.

By making use of the following lemma, found in Müller [8], we reduce the H-type case to the Heisenberg case directly. Recall that for every $\omega \in S^{m-1}$ the map $J_{\omega}$ defines a complex structure on $\mathfrak{v}=\boldsymbol{R}^{2 n}$. Therefore, we can find an orthogonal transformation $\sigma_{\omega}$ such that $J_{\omega}=\sigma_{\omega} J \sigma_{\omega}^{t}$, where $J$ is the $2 n \times 2 n$ matrix defining the standard symplectic form on $\boldsymbol{R}^{2 n}$. We then have the following lemma.

LeMmA 3.2. The mapping $\alpha_{\omega}: N \rightarrow \boldsymbol{H}^{n}$ defined by $\alpha_{\omega}(v, z)=\left(\sigma_{\omega}^{t} v, z \cdot \omega\right),(v, z) \in$ $N$ is an epimorphism of Lie groups. If $k(\omega)$ is the kernel of $\alpha_{\omega}$ and $A=\exp k(\omega)$ then $N / A$ is isomorphic to $\boldsymbol{H}^{n}$. 
Given a lattice subgroup $\Gamma$ of $N$ and $\omega \in S^{m-1}$, let us set $\Gamma_{\omega}=\alpha_{\omega}(\pi(\Gamma))$ where $\pi: N \rightarrow N / A$ is the canonical projection. In order to show that $\Gamma_{\omega}$ is a lattice subgroup of $\boldsymbol{H}^{n}$ we make use of the following theorem from [9].

Theorem 3.3 ([9, Chapter 1, Theorem 4.7]). Suppose that $\Gamma$ is a lattice (resp. uniform lattice) of a locally compact group $G, H$ a closed normal subgroup of $G$ and $\pi: G \rightarrow$ $G / H$ the canonical homomorphism. The subgroup $\pi(\Gamma)$ is a lattice (resp. uniform lattice) in the group $G / H$ if and only if $\Gamma \cap H$ is a lattice (resp. uniform lattice) in the group $H$.

We remark that, in the terminology of the above theorem, $\Gamma$ is said to be a uniform lattice if $G / \Gamma$ is compact. By taking $G=N$ and $H=A$ as in the lemma, we see that $\Gamma_{\omega}$ is a uniform lattice provided $\Gamma \cap A$ is a uniform lattice in the group $A$. To check that this is so, we make use of another theorem from [9].

THEOREM 3.4 ([9, Chapter 1, Theorem 4.5]). Suppose that $\Gamma$ is a discrete subgroup and $H$ a closed subgroup of a locally compact group $G$. Further assume that $\Gamma$ is a uniform lattice of $G$. Then the subgroup $\Gamma \cap H$ is a uniform lattice if and only if the subgroup $H$ is $\Gamma$-closed (i.e., $H \Gamma$ is closed).

Therefore, we only need to show that $A \Gamma$ is closed. This can be done by following the arguments presented in [2, Section 3]. Indeed, we need to start with the representation $\pi_{\lambda, \omega}$ in place of $I_{N}(\phi)$ in [2], identify the kernel of the linear functional $\phi$ and proceed with the computations. We will end up with the problem of showing $A \Gamma$ to be closed. This is precisely the content of [2, Lemma in Section 3]. We refer to this article for the details.

3.4. Heat kernel transform on H-type nilmanifolds. We are ready to look at the heat kernel transform on an H-type nilmanifold $M=\Gamma \backslash N$. We make use of the map $\alpha_{\omega}$ in order to reduce the problem to the Heisenberg nilmanifold $\Gamma_{\omega} \backslash \boldsymbol{H}_{\omega}^{n}$.

First we get a decomposition of $L^{2}(\Gamma \backslash N)$ into subspaces irreducible under the action of $U_{\Gamma}$. From general theory (see Moore [7]), it is known that $L^{2}(\Gamma \backslash N)$ decomposes into a discrete direct sum of irreducible invariant subspaces for $U_{\Gamma}$. On each of these subspaces, $U_{\Gamma}$ will be unitarily equivalent to a finite multiple of $\pi_{\lambda, \omega}$ for some $\lambda>0$ and $\omega \in S^{m-1}$. Let $\pi_{\lambda, \omega}$ be such a representation which occurs in $U_{\Gamma}$ and let $K(\lambda, \omega)$ be the invariant subspace. Recalling that $\pi_{\lambda, \omega}(v, z)=e^{i \lambda z \cdot \omega} \pi_{\lambda, \omega}(v, 0)$, we infer that each function $f \in K(\lambda, \omega)$ is invariant under the right action of $A$ which is just $\exp k(\omega)$. Hence we can think of $K(\lambda, \omega)$ as a subspace of $L^{2}(N / A)$ or even as a subspace of $L^{2}\left(\Gamma_{\omega} \backslash \boldsymbol{H}^{n}\right)$ via the map $\alpha_{\omega}$.

As $K(\lambda, \omega)$ is invariant under $U_{\Gamma}$, the subspace

$$
\tilde{K}(\lambda, \omega)=\left\{f ; f \circ \alpha_{\omega} \circ \pi \in K(\lambda, \omega)\right\}
$$

is invariant under $\Gamma_{\omega}$. Let $\tilde{H}_{\lambda, \omega}^{j}$ be the orthogonal subspaces of $\tilde{K}(\lambda, \omega)$ on each of which $U_{\Gamma_{\omega}}$ is equivalent to $\pi_{\lambda}$. Let $H_{\lambda, \omega}^{j}$ be the pull-back of $\tilde{H}_{\lambda, \omega}^{j}$. Then we have the following theorem.

THEOREM 3.5. Given an H-type nilmanifold $\Gamma \backslash N$, we have

$$
L^{2}(\Gamma \backslash N)=\sum_{\lambda, \omega} \sum_{j} H_{\lambda, \omega}^{j},
$$


where the sum is taken over all $(\lambda, \omega)$ such that $\pi_{\lambda, \omega}$ occurs in $U_{\Gamma}$.

Finally, by combining Theorem 2.9 and the above decomposition, we can describe the image of $L^{2}(\Gamma \backslash N)$ under $S_{t}^{\Gamma}$.

THEOREM 3.6. In above notation, we have

$$
S_{t}^{\Gamma}\left(L^{2}(\Gamma \backslash N)\right)=\sum_{\lambda, \omega} \sum_{j} S_{t}^{\Gamma}\left(H_{\lambda, \omega}^{j}\right) .
$$

In order to describe $S_{t}^{\Gamma}\left(H_{\lambda, \omega}^{j}\right)$, we need to describe $S_{t}^{\Gamma_{\omega}}\left(\tilde{H}_{\lambda, \omega}^{j}\right)$ which we have done in Theorem 2.9 when $\Gamma_{\omega}=\Gamma(\mathbf{l})$. In general there exists $A \in \boldsymbol{A}_{n}$ and $d>0$ such that $\Gamma_{\omega}=A(d . \Gamma(\mathbf{l}))$ and hence an explicit description of $S_{t}^{\Gamma_{\omega}}\left(\tilde{H}_{\lambda, \omega}^{j}\right)$ can, in principle, be written down.

Acknowledgments. This work is supported by J. C. Bose Fellowship from the Department of Science and Technology, Government of India.

\section{REFERENCES}

[ 1 ] L. Auslander And J. Brezin, Translation invariant subspace in $L^{2}$ of a compact nilmanifold I, Invent. Math. 20 (1973), 1-14.

[2] J. BREZIN, Harmonic analysis on nilmanifolds, Trans. Amer. Math. Soc. 150 (1970), 611-618.

[ 3 ] J. CygAn, Heat kernels for class 2 nilpotent groups, Studia Math. 64 (1979), 227-238.

[4] A. KAPLAN AND F. RicCI, Harmonic analysis on groups of Heisenberg type, Harmonic Analysis (Cartona, 1982), 416-435, in Lecture Notes in Mathematics, 992, Springer, Berlin, 1983.

[ 5 ] B. Krötz, S. Thangavelu and Y. XU, The Heat kernel transform for the Heisenberg group, J. Funct. Anal. 225 (2005), 301-336.

[ 6 ] B. KRÖTZ, S. ThAngAVELU AND Y. XU, Heat kernel transform for nilmanifolds associated to the Heisenberg group, Rev. Mat. Iberoamericana. 24 (2008), 243-266.

[ 7 ] C. C. Moore, Decomposition of unitary representations defined by discrete subgroups of nilpotent groups, Ann. of Math. (2) 82 (1965), 146-182.

[ 8 ] D. MÜLLER, Sharp $L^{p}$ estimates for the wave equation on Heisenberg type groups, in Lecture Notes, Orleans, April, 2008.

[9] A. L. OnishchiK AND E. B. Vinberg (Eds.), Lie groups and Lie algebras II, Encyclopaedia Math. Sci. 21, Springer-Verlag, Berlin, 2000.

[10] J. RANDALL, The heat kernel for generalized heisenberg groups, J. Geom. Anal. 6 (1996), 287-316.

[11] F. RICCI, Harmonic analysis on generalized Heisenberg groups, Preprint.

[12] S. Thangavelu, Harmonic analysis on the Heisenberg nilmanifolds, Rev. Un. Mat. Argentina 50 (2009), 75-93.

[13] S. Thangavelu, Gutzmer's formula and the Segal-Bargmann transform, Perspectives in mathematical sciences. II, 209-221, Stat. Sci. Interdiscip. Res., 8, World Sci. Publ., Hackensack, NJ, 2009.

[14] R. Tolimieri, Heisenberg manifolds and theta functions, Trans. Amer. Math. Soc. 239 (1978), 293-319.

DEPARTMENT OF MATHEMATICS

INDIAN INSTITUTE OF SCIENCE

BANGALORE-560 012

INDIA

E-mail address: adgupta@math.iisc.ernet.in, veluma@math.iisc.ernet.in 